Supplement of The Cryosphere, 11, 1015-1033, 2017

http://www.the-cryosphere.net/11/1015/2017/

doi:10.5194/tc-11-1015-2017-supplement

(C) Author(s) 2017. CC Attribution 3.0 License.

(c) (i)

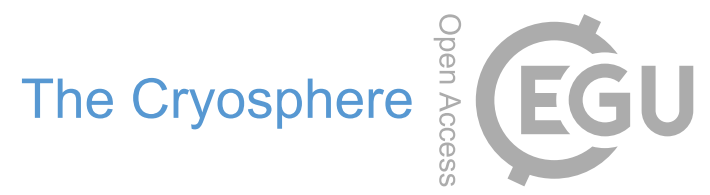

Supplement of

\title{
Reconstructions of the 1900-2015 Greenland ice sheet surface mass balance using the regional climate MAR model
}

Xavier Fettweis et al.

Correspondence to: Xavier Fettweis (xavier.fettweis@ulg.ac.be)

The copyright of individual parts of the supplement might differ from the CC-BY 3.0 licence. 


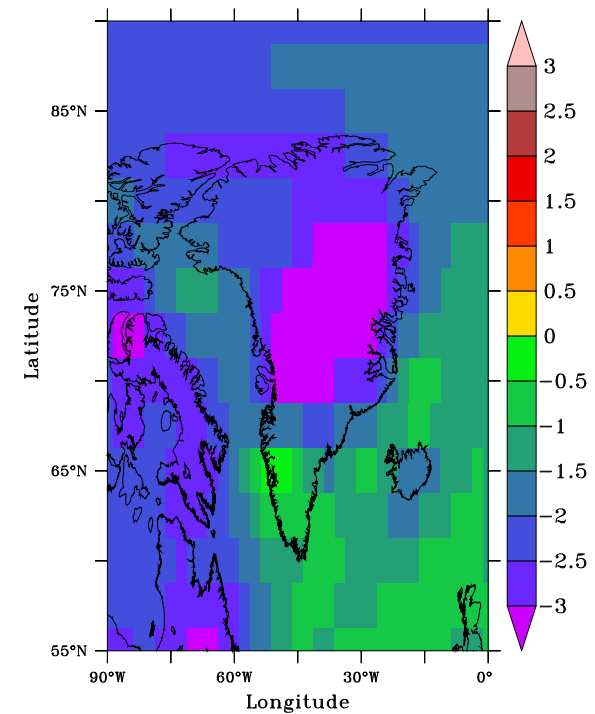

JJA T850: ERA-20c - NCEP1

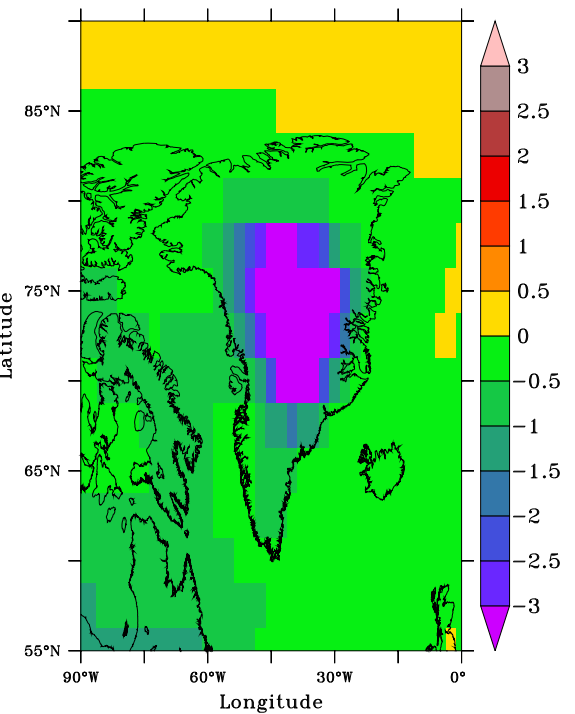

JJA T700: ERA-20c - NCEP1

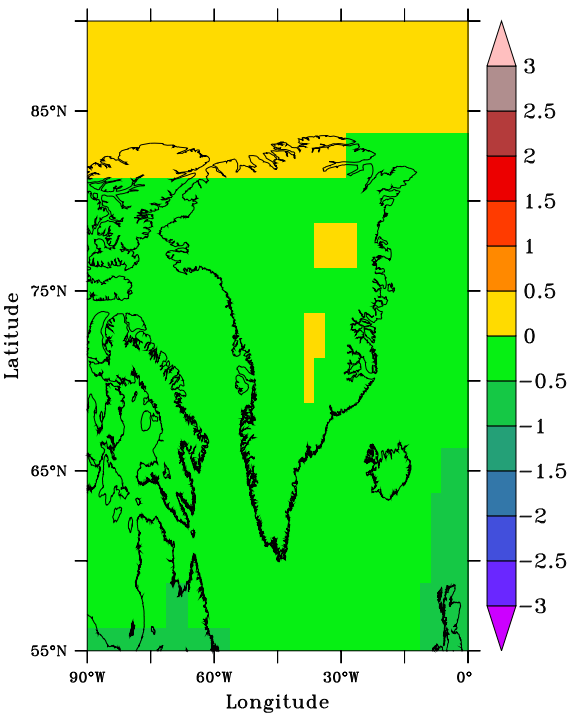

JJA T500: ERA-20c - NCEP1

Figure S1. Differences of the JJA 850, 700 and $500 \mathrm{hPa}$ temperature over 1948-2010 between ERA-20C and NCEPv1. Units are Celsius degrees.

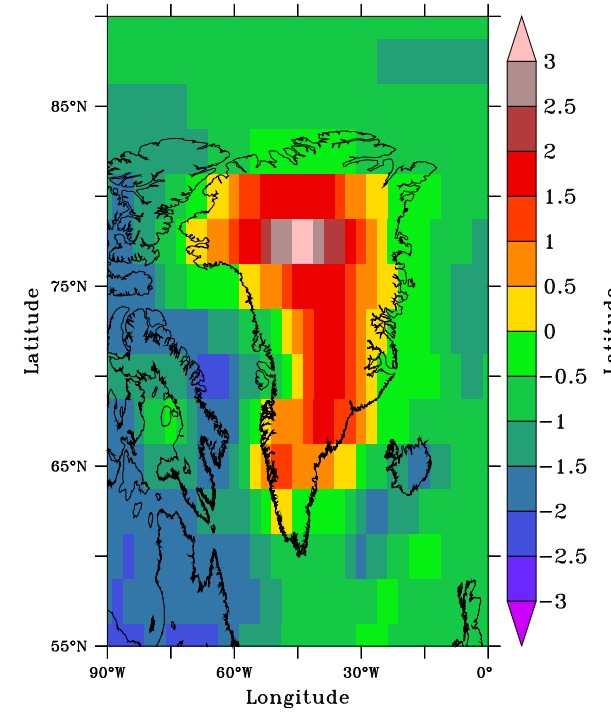

Annual T850: ERA-20c - NCEP1

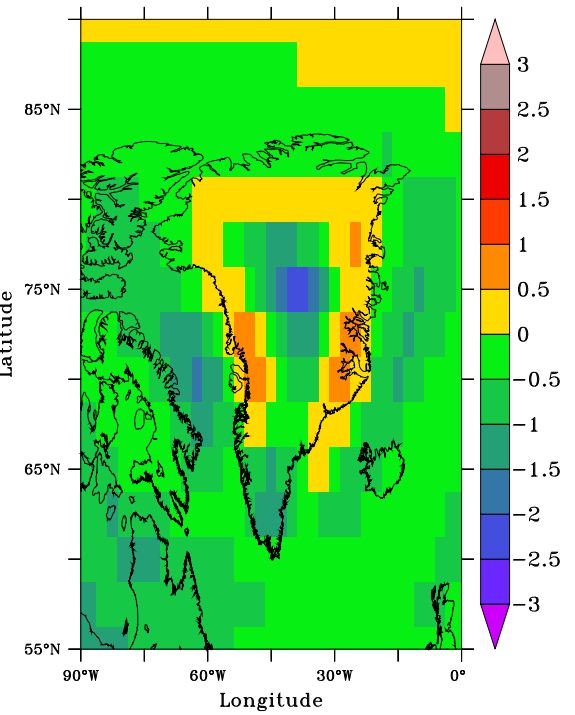

Annual T700: ERA-20c - NCEP1

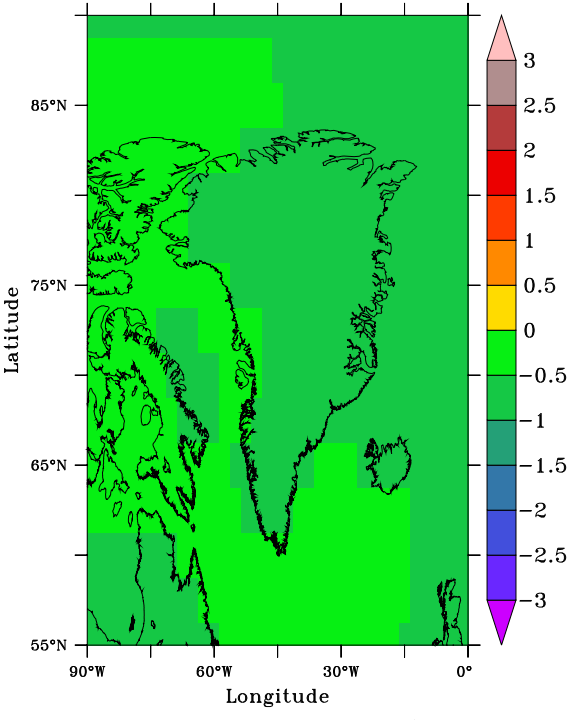

Annual T500: ERA-20c - NCEP1

Figure S2. Differences of the Annual 850, 700 and $500 \mathrm{hPa}$ temperature over 1948-2010 between ERA-20C and NCEPv1. Units are Celsius degrees. 


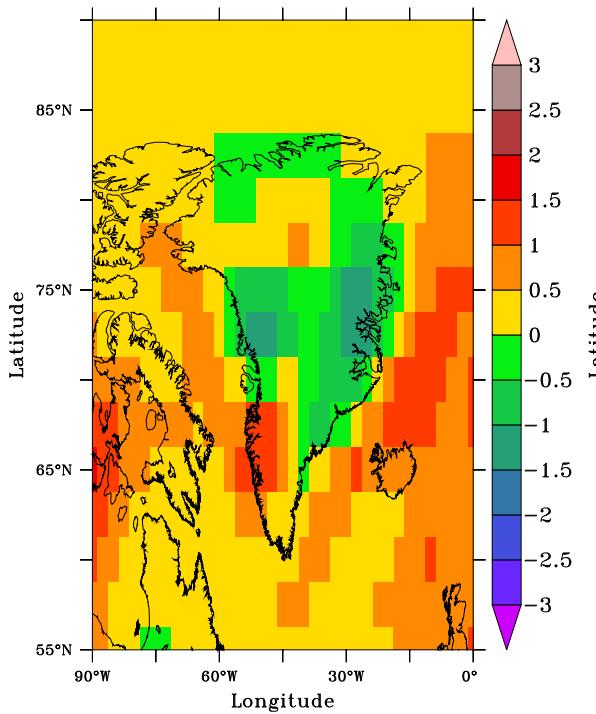

JJA T850: 20CRv2 - NCEP1

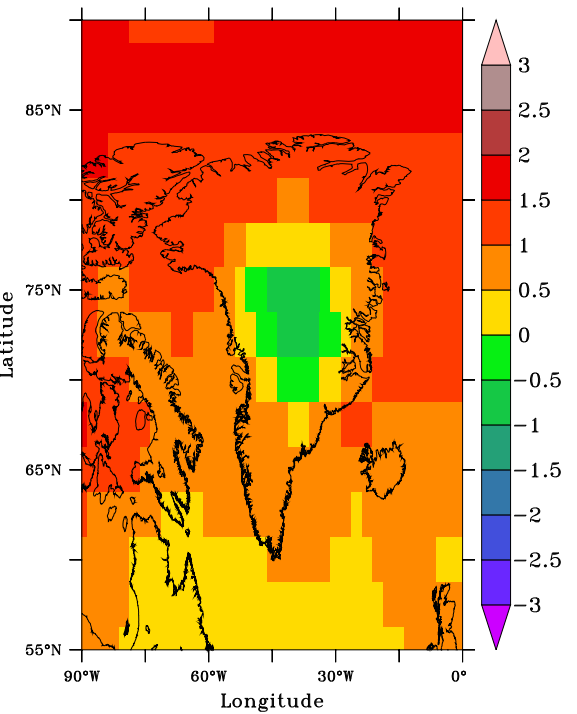

JJA T700: 20CRv2 - NCEP1

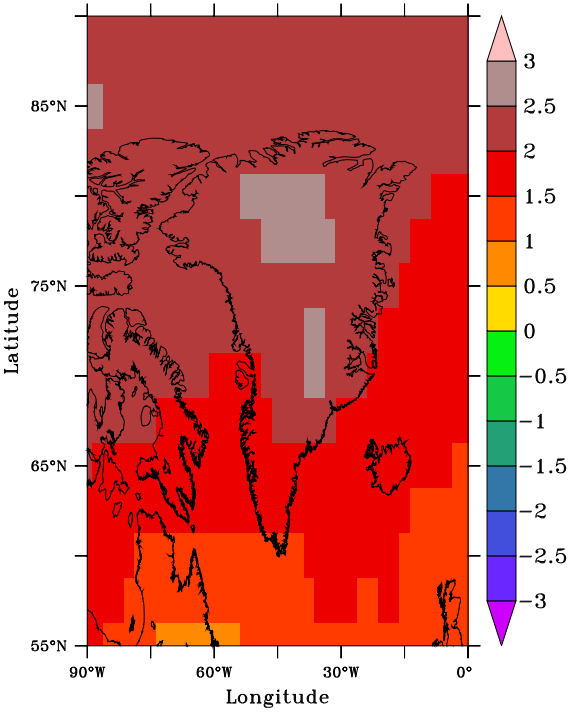

JJA T500: 20CRv2 - NCEP1

Figure S3. Differences of the JJA 850, 700 and $500 \mathrm{hPa}$ temperature over 1948-2010 between 20CRv2 and NCEPv1. Units are Celsius degrees.

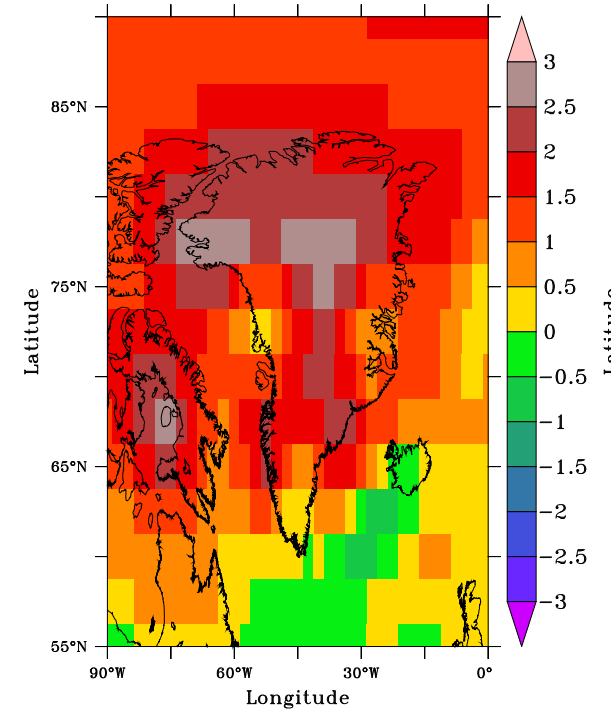

Annual T850: 20CRv2 - NCEP1

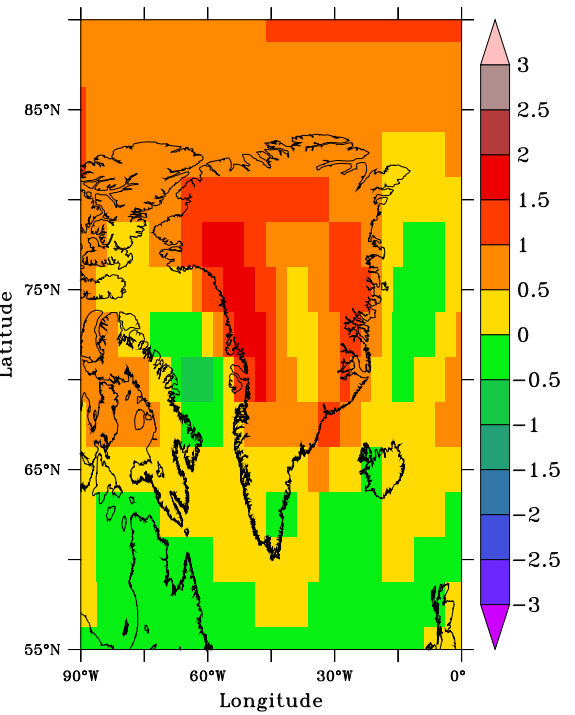

Annual Tr00: 20CRv2 - NCEP1

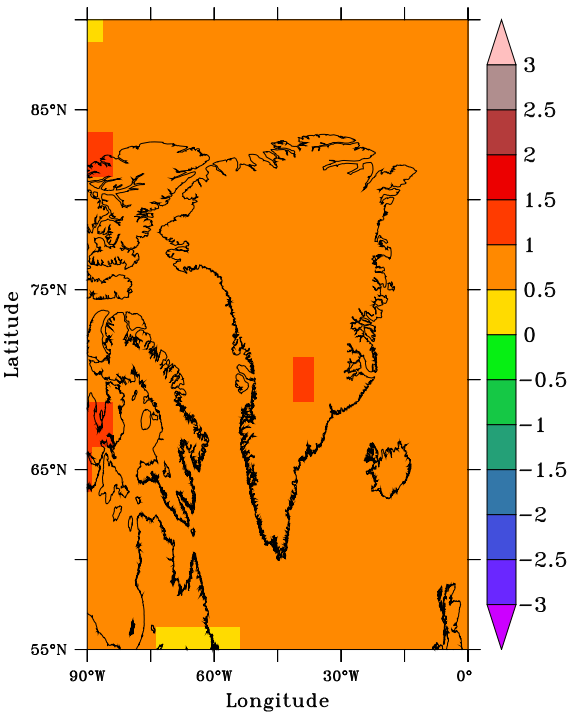

Annual T500: 20CRv2 - NCEP1

Figure S4. Differences of the Annual 850, 700 and 500 hPa temperature over 1948-2010 between 20CRv2 and NCEPv1. Units are Celsius degrees. 


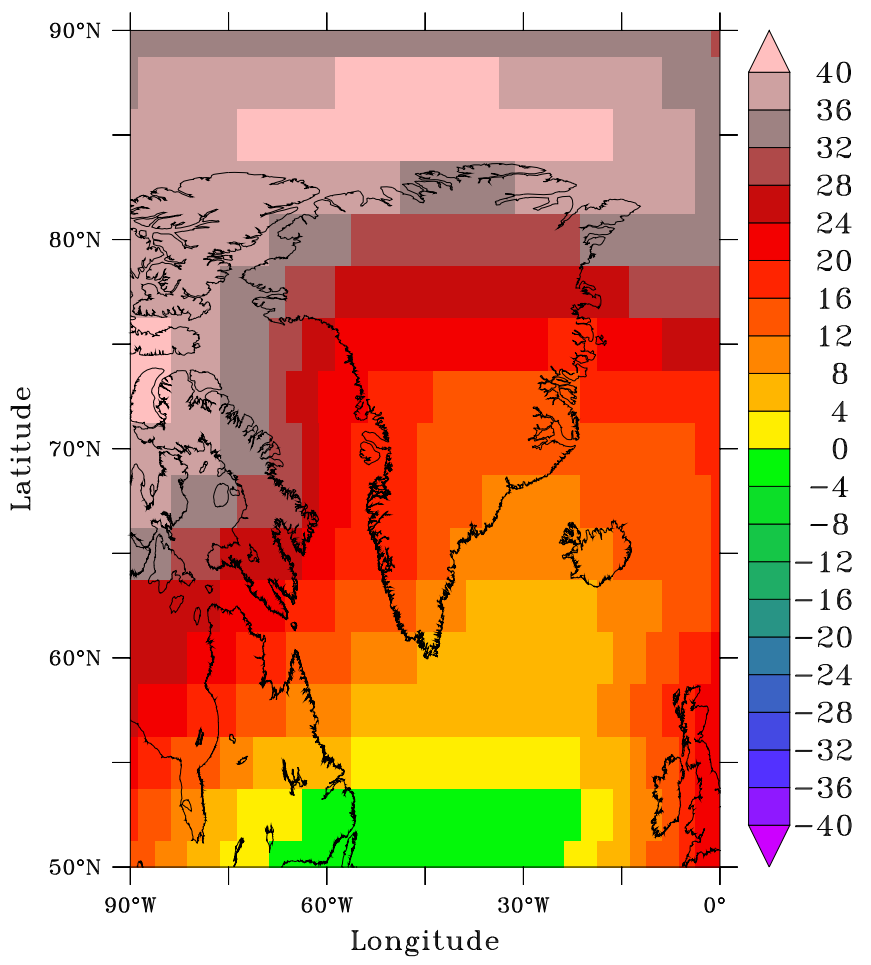

Z500: 20CRv2 - NCEP1 (1948-2010)

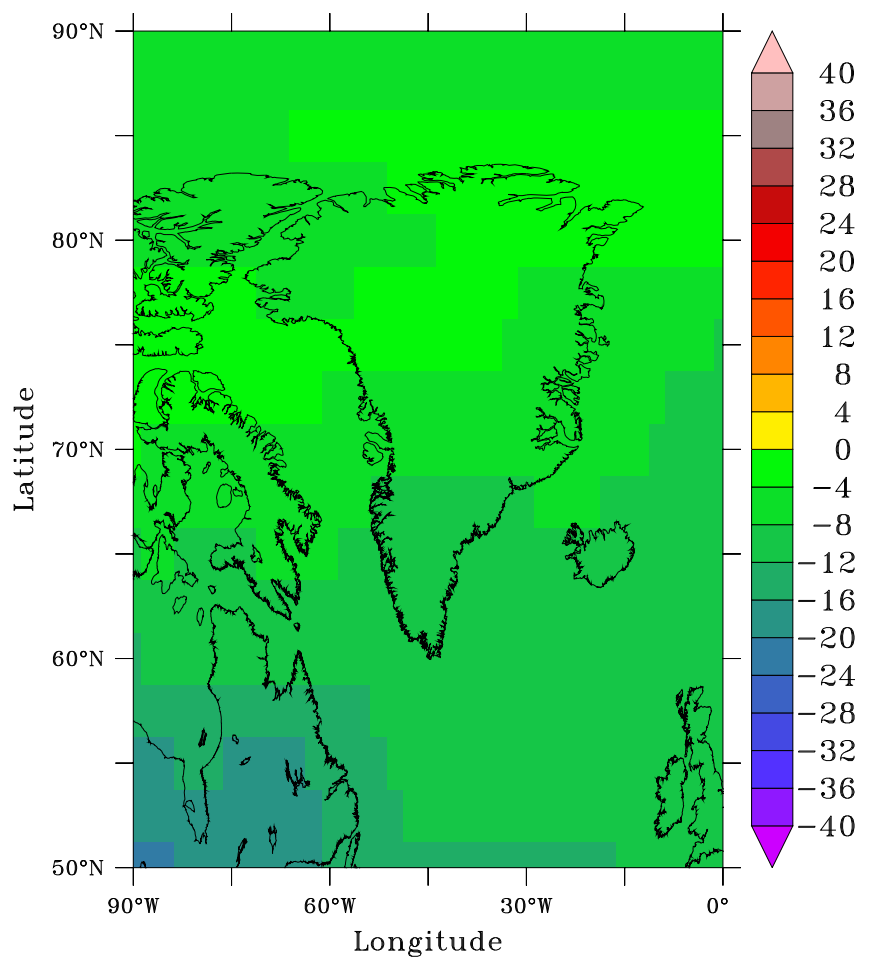

Z500: ERA-20C - NCEP1 (1948-2010)

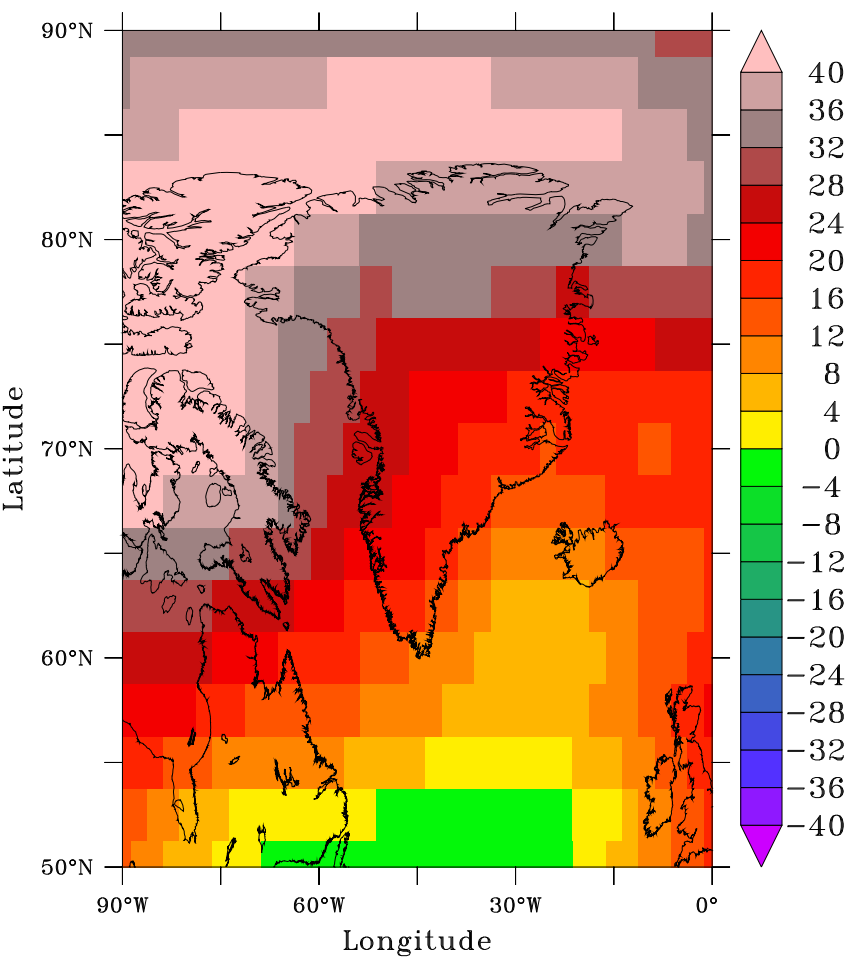

Z500: 20CRv2 - NCEP1 (1980-1999)

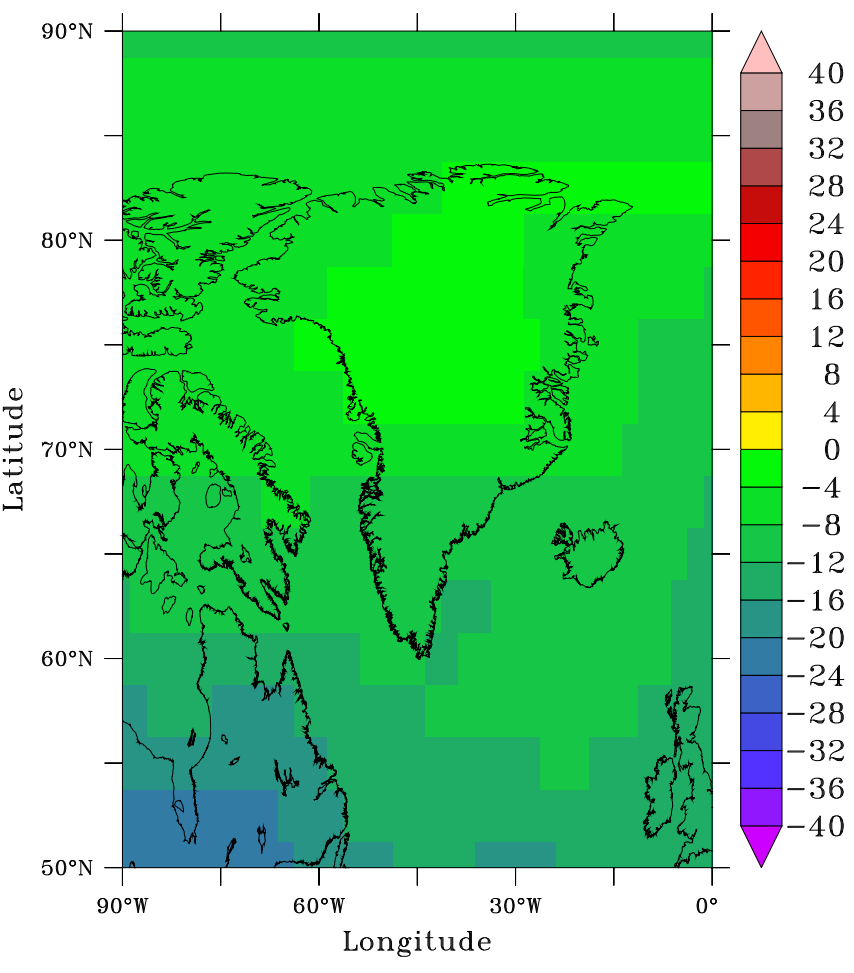

Z500: ERA-20C - NCEP1 (1980-1999)

Figure S5. Differences of the annual Z500 over 1950-2010 and over 1980-1999 between 20CRv2 and NCEPv1. Units are meters. As 20CRv2 (resp. ERA-20c) is too warm (resp. too cold), 20CRv2 (resp. ERA-20c) overestimates (resp. underestimates) the annual geopotential height at $500 \mathrm{hPa}$. However, these biases are constant in time and not impacted by the reference period chosen. 
Table S1. Localisation (Latitude, longitude and elevation) of the 12 AWS's from the PROMICE network used here to validate MAR. The location of the corresponding MAR grid cell is also listed.

\begin{tabular}{lrrrrrr}
\hline AWS & \multicolumn{3}{c}{ PROMICE } & & \multicolumn{3}{c}{ MAR } \\
& Lat. $\left({ }^{\circ} \mathrm{N}\right)$ & Lon. $\left({ }^{\circ} \mathrm{E}\right)$ & Elev. $(\mathrm{m})$ & Lat. $\left({ }^{\circ} \mathrm{N}\right)$ & Lon. $\left({ }^{\circ} \mathrm{E}\right)$ & Elev. $(\mathrm{m})$ \\
\hline KAN_L & 67.10 & -49.93 & 680 & 67.08 & -49.92 & 649 \\
KAN_M & 67.07 & -48.82 & 1270 & 67.08 & -48.78 & 1298 \\
KAN_U & 67.00 & -47.02 & 1850 & 66.98 & -46.93 & 1896 \\
KPC_U & 79.83 & -25.12 & 870 & 79.85 & -25.07 & 766 \\
NUK_L & 64.48 & -49.53 & 560 & 64.49 & -49.54 & 877 \\
NUK_N & 64.95 & -49.88 & 930 & 64.94 & -49.90 & 888 \\
NUK_U & 64.50 & -49.26 & 1140 & 64.50 & -49.26 & 1117 \\
QAS_A & 61.24 & -46.73 & 1009 & 61.30 & -46.75 & 1075 \\
QAS_L & 61.03 & -46.85 & 310 & 61.04 & -46.83 & 525 \\
TAS_L & 65.64 & -38.90 & 270 & 65.64 & -38.91 & 440 \\
TAS_U & 65.70 & -38.87 & 580 & 65.73 & -38.90 & 655 \\
UPE_U & 72.89 & -53.53 & 980 & 72.89 & -53.54 & 1001 \\
\hline
\end{tabular}


Table S2. Same as Table 3 in the manuscript but for each decade over 1910-2010. The numbers of observations (nbr) as well as the standard deviation (std) of observations are also listed.

\begin{tabular}{|c|c|c|c|c|c|c|c|c|c|c|c|}
\hline \multirow[t]{2}{*}{ Decade } & \multirow[t]{2}{*}{ nbr } & \multirow[t]{2}{*}{ std } & \multicolumn{3}{|c|}{ MAR $_{\text {CORR-ERA-20c }}$} & \multicolumn{3}{|c|}{$\mathrm{MAR}_{20 \mathrm{CRv} 2 \mathrm{c}}$} & \multicolumn{3}{|c|}{ BOX13 } \\
\hline & & & BIAS & RMSE & CORR & BIAS & RMSE & CORR & BIAS & RMSE & CORR \\
\hline 1910’s & 12 & 0.73 & 0.03 & 0.20 & 0.97 & -0.02 & 0.18 & 0.97 & 0.16 & 0.35 & 0.95 \\
\hline 1920’s & 19 & 1.13 & 0.03 & 0.41 & 0.94 & -0.02 & 0.29 & 0.97 & 0.66 & 1.04 & 0.84 \\
\hline 1930’s & 27 & 1.13 & -0.04 & 0.23 & 0.98 & -0.22 & 0.43 & 0.97 & 0.43 & 0.83 & 0.98 \\
\hline 1940’s & 45 & 0.11 & -0.01 & 0.04 & 0.92 & -0.03 & 0.05 & 0.90 & -0.03 & 0.05 & 0.93 \\
\hline 1950’s & 274 & 0.68 & 0.10 & 0.45 & 0.77 & -0.13 & 0.55 & 0.76 & 0.11 & 0.49 & 0.78 \\
\hline 1960’s & 107 & 0.49 & 0.16 & 0.39 & 0.71 & 0.12 & 0.40 & 0.78 & 0.02 & 0.32 & 0.78 \\
\hline 1970’s & 162 & 1.07 & -0.11 & 0.35 & 0.96 & -0.17 & 0.40 & 0.97 & 0.39 & 0.64 & 0.88 \\
\hline 1980’s & 1072 & 1.03 & 0.02 & 0.42 & 0.91 & -0.06 & 0.46 & 0.91 & 0.16 & 0.56 & 0.85 \\
\hline 1990’s & 452 & 1.09 & 0.25 & 0.47 & 0.93 & 0.11 & 0.43 & 0.93 & 0.01 & 0.47 & 0.90 \\
\hline 2000's & 210 & 1.52 & 0.08 & 0.64 & 0.91 & -0.12 & 0.62 & 0.92 & 0.28 & 1.16 & 0.68 \\
\hline
\end{tabular}

